Monatsschr Kinderheilkd 2014 [Suppl 2]

162:274-276

DOI 10.1007/s00112-014-3209-3

๑) Springer-Verlag Berlin Heidelberg 2014

\section{Abstracts der Arbeitstagung der AG Pädiatrische Radiologie der Deutschen Röntgengesellschaft (DRG)}

Wissenschaftliche Leitung

Prof. Dr. med. Hans-J. Mentzel, Jena

\section{Kinderradiologie - Fit für den Facharzt}

ZNS

AGPR-SY-FZ-1

Fetale MRT des ZNS

Hirsch F.W. ${ }^{?}$

'Universitätsklinikum Leipzig, Selbstständige Abteilung für Kinderradiologie, Leipzig

Die fetale MRT-Diagnostik als „,second-line imaging“ hat sich als bildgebende Ergänzungsmethode, insbesondere im Bereich der Hirndiagnostik, etabliert. Das MRT erbringt aufgrund des höheren Weichteilkontrastes und der dadurch differenten Gewebeabbildung eine zum Ultraschall oft komplementäre Information.

Für interdisziplinär ausgerichtete Fetalzentren ist diese Mehrinformation des fetalen MRT für bestimmte Indikationen inzwischen unverzichtbar geworden. Vor jeder fetalen MRT-Diagnostik muss ein qualifizierter Ultraschallbefund vorliegen, aus dem sich eine Indikation ableitet und eine Fragestellung an das fetale MRT ergibt. Die Indikationsbreite für das fetale MRT reicht dann von der Frage nach assoziierten Fehlbildungen bis hin zur Planung bei fetaler Chirurgie. Bei einzelnen Krankheitsentitäten, z. B. der Myelomeningocele, hat sich das unmittelbar pränatal durchgeführte MRT als gleichwertig mit der postpartalen Diagnostik herausgestellt. In diesen Fällen ist die In-utero-Untersuchung schonender für das Kind. Auch für die Unterstützung des elterlichen Verständnisses für eine komplexe Pathologie und die nachfolgende interdisziplinäre Beratung kann ein MRT hilfreich sein.

\section{AGPR-SY-FZ-2}

\section{MS, ADEM und weitere entzündliche ZNS-Erkrankungen}

Leclair N. ${ }^{1}$, Sorge I. ${ }^{1}$

'Universitätsklinikum Leipzig, Selbstständige Abteilung für Kinderradiologie, Leipzig

Hintergrund. Das MRT hat die diagnostische Sicherheit der multiplen Sklerose bei Erwachsenen revolutioniert. Mit der Einführung der Mc-Donald-Kriterien 2001 und ihrer Revision 2005 gibt es klare bildgebende Parameter, die im Konsensus mit klinischen und paraklinischen Befunden in den meisten Fällen eine Einordung (in MS, mögliche MS bzw. keine MS) zulassen. Dagegen gibt es im Kindesalter häufiger diagnostische Unsicherheiten, da das MRT-Erscheinungsbild von dem bei Erwachsenen differieren kann. Die Mc-Donald-Kriterien werden bei Kindern oft nicht vollständig erfüllt. Eine Abgrenzung zu anderen demyelinisierenden Erkrankungen wie ADEM ist aufgrund des unterschiedlichen therapeutischen Ansatzes erforderlich.

Methoden. In einer Übersicht sollen die diagnostischen Kriterien einer MS rekapituliert und die Besonderheiten des Kindesalters herausgearbeitet werden. Dabei wird besonderer Wert auf die Differenzierung zwischen dem Klinisch isolierten Syndrom (CIS), ADEM und MS gelegt. Ergebnisse. Im Gegensatz zu den demyelinisierenden Entzündungen ist die Diagnostik von ZNS-Infektionen eine Domäne der Paraklinik. Trotzdem kann die MRT wertvolle Hilfe beim Nachweis von Komplikationen, aber auch in der Erstdiagnostik leisten. Anhand von Bildbeispielen werden typische und seltene Befunde bakterieller, viraler bzw. fungaler zerebraler Infektionen im Kindesalter und ihrer Komplikationen demonstriert.

Lernziele. MR-morphologische Kriterien der MS und die Besonderheiten des Kindesalters, Abgrenzung zu ADEM und Klinisch isoliertem Syndrom (CIS), typische und seltene MRT-Befunde bei bakteriellen, viralen bzw. fungalen ZNS-Infektionen des Kindesalters.

\section{Trauma/Skelett}

\section{AGPR-SY-FT-1}

Bildgebende traumatologische Diagnostik allgemein sowie unter besonderer Berücksichtigung der KADI-Läsionen

Ritter L. ${ }^{1}$

'Universitätsklinikum Leipzig, Selbstständige Abteilung für Kinderradiologie, Leipzig

Die bildgebende traumatologische Diagnostik muss in der Lage sein, lebensbedrohliche Verletzungen schnell zu erkennen. Hierfür sind Ultraschall und CT die führenden Methoden.

Außerdem müssen vor allem Frakturen mit Einschluss der für das Wachstum des Kindes hauptverantwortlichen Teile des Skelettsystems zuverlässig diagnostiziert werden. Hierbei sind die sogenannten KADILäsionen von besonderer Bedeutung. Drei dieser Verletzungen betreffen allein das Ellenbogengelenk. Deshalb liegt ein Fokus des Vortrages auf dieser Region bzw. diesen Läsionen.

Die Komplexität des kindlichen Traumas sowie die zur Verfügung stehenden diagnostischen Modalitäten mit ihren Vor- und Nachteilen erfordern den differenzierten Einsatz dieser Untersuchungsverfahren. Im Rahmen des Vortrages wird auch auf diese Thematik eingegangen. 


\section{AGPR-SY-FT-2}

\section{Bildgebende Diagnostik bei Rückenschmerzen im Kindesalter}

Stegmann J.', Leenen A. ', Tholen P.'

'Kinderkrankenhaus Wilhelmstift, Hamburg, Abt. für Bildgebende Diagnostik, Hamburg

Hintergrund. Rückenschmerzen im Kindesalter sind seltener als bei Erwachsenen; häufiger als bei diesen sind sie jedoch Ausdruck einer ernsthaften Erkrankung. Daher sollte bei diesem Symptom eine eingehende und gründliche Diagnostik erfolgen.

Methoden. In Abhängigkeit von der Anamnese, insbesondere der Tatsache, ob ein Trauma vorausgegangen ist oder nicht, sowie vom Alter der Patienten werden Beispiele aus der bildgebenden Diagnostik vorgestellt. Dabei wird besonders auf die zielführende Auswahl der bildgebenden Methoden eingegangen. Auf die vorhandenen Leitlinien für die Abklärung nichttraumatischer Rückenschmerzen wird eingegangen. Ergebnisse und Schlussfolgerung. Die Auswahl der bildgebenden Methoden richtet sich - wie immer im Kindesalter - einerseits danach, welche von ihnen auf dem schnellsten Weg und mit möglichst geringer Strahlenexposition zum Ziel (Diagnosestellung bzw. -ausschluss) führen. Insbesondere wird dargestellt, welchen Wert der Röntgendiagnostik und der Kernspintomographie dabei zukommt. Anhand der Beispiele werden die Schlussfolgerungen erläutert.

\section{AGPR-SY-FT-3}

\section{Tumoren und "tumor-like lesions" am Skelett}

\section{Steinborn M.}

'Städtisches Klinikum München-Schwabing, Institut für Diagnostische und Interventionelle Radiologie und Kinderradiologie, München

Hintergrund. Tumoren und "tumor-like lesions" des Knochens können als Zufallsbefunde gefunden werden oder durch Schmerzen, Schwellung oder pathologische Fraktur symptomatisch werden. Lokalisation, Alter des Patienten und Anamnese geben häufig bereits wichtige Hinweise auf mögliche Differenzialdiagnosen.

Methoden. Die initiale radiologische Abklärung erfolgt gewöhnlich in Form eines konventionellen Röntgenbildes in 2 Ebenen. Die Beurteilung eines Knochentumors im konventionellen Röntgenbild sollte insbesondere das Destruktionsmuster und die Periostreaktion beschreiben, wodurch wichtige Informationen über die Aggressivität und damit Dignität einer Läsion gewonnen werden. Das Destruktionsmuster kann dabei nach der Lodwick-Klassifikation eingeteilt werden, welche den Destruktionstyp, die Begrenzung und den Bezug der Läsion zur Kortikalis berücksichtigt. Die Beurteilung der Periostreaktion gibt ebenfalls Hinweise auf Aggressivität und Wachstumsgeschwindigkeit einer Läsion, wobei kontinuierliche, unterbrochene und komplexe Formen unterschieden werden. Zusätzlich gibt die Tumormatrix wichtige differenzialdiagnostische Hinweise.

Schlussfolgerung. Aufgrund der großen Bedeutung der röntgenologischen Analyse einer Läsion ist häufig auch eine CT-Untersuchung zur genaueren und überlagerungsfreien Darstellung hilfreich. Handelt es sich aufgrund der Röntgenuntersuchung nicht eindeutig um eine benigne Läsion wird im nächsten Schritt in der Regel eine MRT angeschlossen. Die MRT dient der genauen Darstellung der Tumorausdehnung und insbesondere der Darstellung des Weichteilanteils. Die MRT sollte auch großzügig zum Ausschluss von Tumoren in radiologisch schwer beurteilbaren Regionen eingesetzt werden (Becken, Wirbelsäule).

\section{MRT/Strahlenschutz}

\section{AGPR-SY-FM-1}

\section{Ganzkörper-MRT bei Skeletterkrankungen}

\section{Mentzel HJ, Stenzel M}

Universitätsklinikum Jena, Sektion Pädiatrische Radiologie, Institut für Diagnostische und Interventionelle Radiologie

Die Ganzkörper-MRT stellt aufgrund der technischen Errungenschaften in der MRT-Technik auch im Kindesalter bei speziellen Fragestellungen zunehmend eine mögliche Alternative zur CT und zur Nuklearmedizin dar. Ziel des Beitrags ist es, neben den technischen Voraussetzungen die Durchführung der Ganzkörper-MRT aufzuzeigen. Es sollen fragestellungsbezogene Protokolle gezeigt und anhand von Beispielen auf Indikationen für die GK-MRT eingegangen werden. Die Bedeutung der GK-MRT für entzündlich rheumatische Erkrankungen oder für onkologische Fragestellungen wird dargestellt, auf Grenzen der Methode verwiesen.

\section{AGPR-SY-FM-2}

\section{PET/MRT im Kindesalter}

Sorge I. ${ }^{,}$Hirsch W. ${ }^{1}$

${ }^{1}$ Universitätsklinikum Leipzig, Selbstständige Abteilung für Kinderradiologie, Leipzig

Seit 2011 wird in Leipzig das Ganzkörper-PET/MRT (mMR, SIEMENS/ Germany) routinemäßig in der Diagnostik und bei Verlaufskontrollen bei Kindern mit disseminierten onkologischen Erkrankungen eingesetzt. Es handelt sich um das erste Gerät mit CE-Zertifizierung, so dass wir inzwischen auf eine dreijährige klinische Erfahrung mit der Methodik - und damit die weltweit größte untersuchte Kindergruppe zurückblicken können.

Durch das PET/MRT wurde in Leipzig das PET/CT bei Kindern komplett abgelöst. Das PET/MRT ersetzt die Strahlenexposition des CT-Anteiles, der bei herkömmlichen PET/CT notwendig war. Die effektive Dosis eines PET/MRT liegt bei nur noch 20\% im Vergleich zu einem vergleichbaren PET/CT.

Die zeitgleiche, simultane Datenakquisition von PET und MRT kombiniert die Vorteile der beiden komplementären, bisher getrennten Modalitäten. Das PET kann die Stoffwechselaktivität der Tumorläsionen und damit die biologische Aktivität messen. Das MRT addiert strukturelle und anatomische Informationen. Darüber hinaus erlaubt die Ganzkörper-Diffusionsbildgebung Aussagen über die biologische Zellularität von Tumoren. Alle diese Informationen sind damit in einer "one-stopshop"-Diagnostik zu erhalten, die in der Primärdiagnostik und insbesondere im Restaging von kindlichen Tumoren Zeit und Ressourcen spart.

Ein Problem ist die relativ lange Untersuchungszeit der PET/MRT, die Untersuchungsprotokolle müssen entsprechend angepasst werden.

Anhand von typischen Fallbeispielen aus dem Bereich GanzkörperPET-MRT und Hirn-PET-MRT berichten wir über unsere Erfahrungen mit der neuen Hybridbildgebung. Die PET/MRT erweist sich bei den bisher untersuchten Kindern als unerwartet stabile, zuverlässige Bildgebungsmodalität, welche einen diagnostischen Mehrwert generiert.

\section{AGPR-SY-FM-3 \\ Strahlenexposition und Strahlenschutz in der Kinderradiologie}

Strocka S. ${ }^{1}$

'Universitätsklinikum Leipzig, Selbstständige Abteilung für Kinderradiologie, Leipzig

Kindliches Gewebe weist im Vergleich mit Erwachsenen eine höhere Strahlenempfindlichkeit auf, daher ist in dieser Altersgruppe ein be- 
sonders restriktiver Umgang mit ionisierender Strahlung notwendig. Strahleninduzierte Malignome werden mit hoher Wahrscheinlichkeit erlebt und Kinder haben als potenzielle Eltern ein zusätzliches genetisches Strahlenrisiko. Bei der Wahl der geeigneten Diagnostik sind alternativen Verfahren wie MRT und Sonographie daher ein besonders hoher Stellenwert im klinischen Alltag einzuräumen und, wenn immer sinnvoll möglich, gegenüber der Röntgendiagnostik zu präferieren. Ist der Einsatz ionisierender Strahlung unumgänglich, so bedarf es neben speziell geschultem Personal auch adaptierter Untersuchungstechniken und -protokolle, die dem Alter und der klinischen Fragestellung angepasst sind, um die Strahlenexposition so gering wie möglich zu halten bei gleichzeitiger Gewährleistung der diagnostischen Sicherheit.

\section{Abdomen}

\section{AGPR-SY-FA-1}

\section{Akutes Abdomen im Kindesalter}

\section{Stenzel M. ', Mentzel $\mathrm{HJ}^{\prime}$}

${ }^{1}$ Institut für Diagnostische und Interventionelle Radiologie, Sektion Pädiatrische Radiologie, Universitätsklinikum der Friedrich-Schiller-Universität Jena, Jena

Das akute Abdomen stellt einen Notfall dar, der neben klinischen und laborchemischen Untersuchungen oft eine zügige und adäquate Bildgebung zur Diagnosefindung notwendig macht. Die Wahl der bildgebenden Methode ist abhängig von Alter und Kooperationsfähigkeit des Kindes und der vermuteten Pathologie. Im Gegensatz zum Erwachsenen spielt die Computertomographie bei Kindern eine untergeordnete Rolle, da die Krankheitsbilder erheblich abweichen und strahlensparende Verfahren in Deutschland/Europa bevorzugt eingesetzt werden. Fallbasiert werden die häufigen Ursachen eines akuten Abdomen im Kindesalter diskutiert: nekrotisierende Enterokolitis, Darmatresien, -duplikaturen, hypertrophe Pylorusstenose, Appendizitis, chronischentzündliche Darmerkrankungen, Pankreatitis. In die Diskussion eingehen sollen die Aspekte der Sensitivität/Spezifität der Untersuchungen, deren Verfügbarkeit und die Möglichkeiten der Dokumentation.

\section{AGPR-SY-FA-2}

\section{Abdomen-MRT - pädiatrische Besonderheiten}

\author{
Hirsch F.W. \\ 'Universitätsklinikum Leipzig, Selbstständige Abteilung für Kinderradio- \\ logie, Leipzig
}

Sowohl die Fragestellung als auch die MRT-Untersuchungstechnik unterscheidet sich - insbesondere bei kleinen Kindern und Säuglingen - oft deutlich von der Abdomenuntersuchung im Erwachsenenalter. Eine gute Untersuchungsvorbereitung, eine adäquate Atemtriggerung und eine fragestellungsadaptierte Untersuchungsstrategie sind der Schlüssel zum Erfolg.

Da es keine einheitlichen und verbindlichen Protokolle bei kindlichen MR-Untersuchungen gibt und weil daher die Untersuchungsstrategie oft von Einrichtung zu Einrichtung variiert, wird das Untersuchungsprozedere der Leipziger Kinderradiologie bei abdominellen Fragestellungen erläutert. Neben der Darstellung des Vorgehens bei üblichen Standardfragestellungen wird auch auf folgende Besonderheiten der kinderradiologischen MR-Praxis im Abdominalbereich eingegangen: MR-Cholangiographie, MR-Angiographie, Eisenbelastung der Leber, Fettquantifizierung des Abdomens/der Leber und der Einsatz von Diffusionssequenzen.

\section{AGPR-SY-FA-3 \\ MRT des Magen-Darm-Trakts}

Mentzel HJ, Stenzel M

Universitätsklinikum Jena, Sektion Pädiatrische Radiologie, Institut für Diagnostische und Interventionelle Radiologie

Die MRT des Darms ist mittlerweile Gegenstand von Leitlinien zur Diagnostik bei Chronisch entzündlichen Darmerkrankungen im Kindes- und Jugendalter. Ziel des Beitrags ist es, die verschiedenen Möglichkeiten der Darm-MRT sowie die Vor- und Nachteile der Methoden aufzuzeigen. Neben der konventionellen MRT des Abdomens, bei der die Darmschlingen mit beurteilt werden können, gibt es dedizierte Darm-MRT-Untersuchungen mit Füllung des Darmtrakts. Dabei kommen unterschiedliche Flüssigkeiten zum Einsatz. Die Applikation erfolgt entweder oral (Hydro-MRT) oder über eine nasojejunale Sonde (Sellink-MRT). Die Applikation von Kontrastmittel ist bislang essentiell zur Beurteilung der Aktivität entzündlicher Veränderungen und zum Aufzeigen von Komplikationen. Neuere Techniken wie die Diffusionswichtung könnten perspektivisch als Alternative zur CE-MRT eingesetzt werden. 